\title{
Qualidade de Vida de Pacientes com Câncer Colorretal em Uso de Suplementação Dietética com Fungos Agaricus Sylvaticus após Seis Meses de Segmento: Ensaio Clínico Aleatorizado e Placebo-Controlado
}

\author{
Quality of Life of Patients with Colorectal Cancer on Dietary Supplementation \\ with Agaricus Sylvaticus Fungus: After Six Months of Segment: Randomized and \\ Placebo-Controlled Clinical Trial
}

\author{
RENATACOSTAFORTES ${ }^{1}$; VIVIANE LACORTE RECÔVA²; ANDRESA LIMA MELO²; \\ MARIA RITA CARVALHO GARBINOVAES ${ }^{3}$
}

\begin{abstract}
${ }^{1}$ Universidade de Brasília (DF)/ Faculdade de Ciências e Educação Sena Aires (GO); ${ }^{2}$ Faculdade de Medicina da Escola Superior em Ciências da Saúde do Distrito Federal/ ESCS/ FEPECS (DF); ${ }^{3}$ Universidade de Brasília (DF)/ Faculdade de Medicina da Escola Superior em Ciências da Saúde do Distrito Federal ESCS/ FEPECS (DF).
\end{abstract}

FORTES RC; RECÔVA VL; MELO AL; NOVAES MRCG. Qualidade de Vida de Pacientes com Câncer Colorretal em Uso de Suplementação Dietética com Fungos Agaricus Sylvaticus após Seis Meses de Segmento: Ensaio Clínico Aleatorizado e Placebo-Controlado Rev bras Coloproct, 2007;27(2): 130-138.

RESUMO: Introdução: $O$ câncer gastrointestinal compromete a qualidade de vida devido às alterações fisiológicas, metabólicas e psicológicas. Fungos medicinais podem melhorar a qualidade de vida de pacientes com câncer. Objetivo: Avaliar os efeitos da suplementação dietética com fungos Agaricus sylvaticus na qualidade de vida de pacientes com câncer colorretal em fase pósoperatória. Metodologia: Ensaio clínico randomizado, duplo-cego, placebo-controlado, realizado no Hospital de Base do Distrito Federal. Amostra constituída por 56 pacientes ( 24 homens e 32 mulheres), estádios I, II e III, separados em grupos placebo e Agaricus sylvaticus (30 $\mathrm{mg} / \mathrm{kg} / \mathrm{dia})$, e acompanhados por um período de seis meses. Para avaliar os indicadores da qualidade de vida foram utilizados um formulário-padrão e uma anamnese dirigida-padrão. Os resultados foram analisados de forma qualitativa e descritiva, utilizando os programas Microsoft Excel 2003 e Epi Info 2004. Resultados: Após seis meses de tratamento, observou-se, no grupo Agaricus sylvaticus, aumento da adesão à prática de atividade física, melhora da disposição e do humor, redução das queixas de dores e das alterações do sono como insônia e noites mal dormidas comparado com o grupo placebo. Conclusão: Os resultados sugerem que a suplementação dietética com Agaricus sylvaticus pode melhorar a qualidade de vida de pacientes no pósoperatório de câncer colorretal.

Palavras-chave: Fungos Agaricus sylvaticus, qualidade de vida, câncer colorretal, alterações metabólicas, alterações fisiológicas.

\section{INTRODUÇÃO}

A qualidade de vida é definida pela Organização Mundial de Saúde como "a percepção do indivíduo de sua posição na vida, no contexto da cultura e sistema de valores nos quais ele vive e em relação aos seus objetivos, expectativas, padrões e preocupações".

O câncer é capaz de alterar significativamente a qualidade de vida dos pacientes através da manifes-

Trabalho atribuído ao Departamento de Pós-Graduação em Nutrição Humana, Faculdade de Ciências da Saúde, Universidade de Brasília, DF. Realizado no Hospital de Base da Secretaria de Estado de Saúde do Distrito Federal, Brasília, DF. Baseado em dissertação (mestrado) sob o tema "Avaliação da suplementação nutricional com cogumelos Agaricaceae em portadores de câncer gastrointestinal", Universidade de Brasília, ano de defesa: 2007, 200 páginas. Não há conflito de interesses. 
tação de diversas alterações de humor, motoras, somáticas, sociais, cognitivas, dentre outras ${ }^{2}$.

Substâncias bioativas com propriedades farmacológicas como glucanas, proteoglucanas, lectinas, ergosterol e arginina têm sido identificadas e isoladas em numerosas espécies de fungos medicinais ${ }^{3,4}$.

Os fungos também apresentam importante valor nutricional devido ao elevado conteúdo de fibras, proteínas de alto valor biológico, ácidos graxos insaturados, além de quantidades apreciáveis de vitaminas e minerais ${ }^{3-6}$.

Evidências científicas têm demonstrado que fungos Agaricaceae podem aumentar a sobrevida e melhorar a qualidade de vida com o mínimo de efeitos adversos em pacientes com neoplasias malignas ${ }^{3,5-8}$.

O objetivo deste estudo foi avaliar os efeitos da suplementação dietética com fungos Agaricus sylvaticus em pacientes no pós-operatório de câncer colorretal, após seis meses de tratamento, a respeito dos indicadores da qualidade de vida - sedentarismo, tabagismo, etilismo, distúrbios do sono, alterações na disposição e no humor e presença de dores - que acometem principalmente os pacientes com câncer.

\section{MATERIAIS E MÉTODOS}

\section{Metodologia do estudo}

$\mathrm{O}$ estudo foi conduzido mediante um ensaio clínico randomizado, duplo-cego, placebo-controlado, com alocação aleatória dos pacientes nos grupos de estudo. Foi aprovado pelo Comitê de Ética em Pesquisa da Secretaria de Estado de Saúde do Distrito Federal, sob o protocolo $\mathrm{n}^{\circ} 051 / 2004$. O termo de consentimento livre e esclarecido foi obtido dos pacientes, cuja participação foi voluntária. O trabalho foi desenvolvido no Ambulatório de Proctologia do Hospital de Base do Distrito Federal, Brasil, no período de novembro de 2004 a julho de 2006.

\section{Amostra}

A amostra foi constituída por 56 pacientes ( 24 homens e 32 mulheres) com câncer colorretal, estádios I $(n=12)$, II $(n=16)$ e III $(n=28)$, separados em dois grupos: placebo e suplementado com fungo Agaricus sylvaticus.

Os pacientes foram selecionados de acordo com os seguintes critérios de inclusão: portadores de câncer colorretal em fase pós-operatória, de três meses a dois anos de intervenção cirúrgica, idade superi- or a vinte anos; e de exclusão: gestantes, lactantes, acamados, deficientes físicos, pacientes em uso de terapia alternativa, portadores de outras doenças crônicas não-transmissíveis e em processo de metástase.

\section{Extrato com fungos Agaricus sylvaticus}

O fungo Agaricus sylvaticus, cujo nome popular é Cogumelo do Sol, foi obtido de um produtor, credenciado pela Empresa Brasileira de Agropecuária - Embrapa, da região de Tapiraí, interior do Estado de São Paulo. O extrato do fungo foi obtido por imersão do material desidratado em água quente por $30 \mathrm{minu}-$ tos, liquidificado, peneirado e seco em dissecador. A análise da composição do Agaricus sylvaticus foi realizada pelo Japan Food Research Laboratories Center e revelou a presença de carboidratos $(18,51 \mathrm{~g} /$ $100 \mathrm{~g})$, lipídeos $(0,04 \mathrm{~g} / 100 \mathrm{~g})$, ergosterol $(624 \mathrm{mg} / 100 \mathrm{~g})$, proteínas $(4,99 \mathrm{~g} / 100 \mathrm{~g})$, aminoácidos (arginina-1,14\%; lisina-1,23\%; histidina- $0,51 \%$, fenilalanina- $0,92 \%$, tirosina- $0,67 \%$, leucina- $1,43 \%$, metionina- $0,32 \%$, valina$1,03 \%$, alanina- $1,28 \%$, glicina- $0,94 \%$, prolina- $0,95 \%$, ácido glutâmico-3,93\%, serina- $0,96 \%$, treonina- $0,96 \%$, ácido aspártico- $1,81 \%$, triptofano- $0,32 \%$, cisteína$0,25 \%$ ) e micronutrientes em quantidades-traço.

O extrato seco foi transformado em comprimidos, seguindo procedimento farmacotécnico. A dosagem do fungo administrada aos pacientes do grupo suplementado foi equivalente a $30 \mathrm{mg} / \mathrm{kg} / \mathrm{dia}$, fracionada em duas tomadas diárias. Ao grupo de pacientes que recebeu o placebo, foram administrados os comprimidos nas mesmas quantidades, com os mesmos excipientes e valor calórico, porém sem o extrato do Agaricus sylvaticus. Todos os pacientes ingeriram seis comprimidos por dia (três pela manhã e três à tarde, nos intervalos entre as refeições) durante um período de seis meses.

\section{Evolução clínica}

O período de acompanhamento dos pacientes foi de seis meses, sendo que nos três primeiros meses foram realizadas consultas quinzenais para a avaliação clínica e, nos últimos três meses, as consultas passaram a ser a cada 30 dias.

Os pacientes permaneceram com dieta habitual, embora durante o tratamento tenham recebido orientações gerais sobre como manter uma alimentação saudável. Após seis meses de acompanhamento, na alta hospitalar, foi indicada dieta individualizada para todos os pacientes e os mesmos foram encaminhados 
a outros profissionais da área de saúde, quando necessário.

Todos os pacientes foram contactados pelos pesquisadores, semanalmente, via telefone, para esclarecimentos de dúvidas, verificação do uso adequado do cogumelo segundo as orientações e confirmação do agendamento, garantindo maior adesão ao tratamento e controle sobre a continuidade do estudo.

Foram considerados desistentes os pacientes que compareceram somente às primeiras consultas $\mathrm{e}$ os que não vieram às consultas durante o período de seis meses. Aqueles que faleceram antes do final do tratamento não foram incluídos na amostragem.

\section{Avaliação de alguns indicadores da quali- dade de vida}

Utilizou-se um formulário-padrão e uma anamnese dirigida-padrão para avaliar alguns indicadores da qualidade de vida. $\mathrm{O}$ formulário foi aplicado no primeiro dia da consulta e, nas consultas posteriores, aplicou-se a anamnese dirigida. Toda a coleta de dados foi realizada por pesquisadores previamente treinados.

Os hábitos considerados como componentes do estilo de vida foram sedentarismo, tabagismo, etilismo, alterações do sono, alterações na disposição, humor e dor. Quanto ao sedentarismo, foram considerados sedentários os pacientes que não apresentavam algum tipo de atividade física, pelo menos uma vez por semana por, no mínimo, 30 minutos. Em relação ao tabagismo, os pacientes foram classificados como tabagistas, ex-tabagistas e não-tabagistas (nunca fumaram). Quanto ao etilismo, foram caracterizados como etilistas e não-etilistas. Com relação às alterações do sono, os pacientes foram avaliados de acordo com a presença de insônia e noites mal dormidas. Em relação à disposição, os pacientes foram classificados em bem dispostos e cansados/fadigados. Todos os pacientes foram avaliados, através de imagens simbólicas, em feliz/satisfeito (J), nem feliz nem triste (K), infeliz/insatisfeito (L) para classificação do humor. Quanto à presença de queixas de dores, as mesmas foram classificadas em cefaléia, dor retal, dor abdominal, dor no local da cirurgia e outras.

Todos os indicadores de qualidade de vida foram avaliados em três momentos distintos: antes do início do tratamento, com três meses e, após seis meses, exceto para o humor cujos dados foram analisados no início e final da pesquisa.

\section{Análise estatística}

Os pacientes foram separados em grupos placebo e Agaricus sylvaticus para a comparação dos resultados. Todos os dados coletados foram analisados de forma qualitativa e descritiva, utilizando os programas Microsoft Excel 2003 e Epi Info 2004 para Windows, versão 3.3.2.

\section{RESULTADOS}

Após seis meses de segmento, 56 pacientes com câncer colorretal concluíram o estudo, sendo 32 mulheres $(57,1 \%)$ e 24 homens $(42,9 \%)$, separados em grupos placebo e Agaricus sylvaticus (tabela 1).

Os pacientes do grupo placebo $(n=28)$ tinham idade média de 59,14 $\pm 12,95$ anos. Com relação ao gênero, $57,1 \%(n=16)$ eram do sexo feminino, e $42,9 \%$ $(n=12)$ do sexo masculino.

Os pacientes do grupo que recebeu Agaricus sylvaticus $(n=28)$ tinham idade média de 56,34 $\pm 15,53$ anos. Quanto ao gênero, $57,1 \%(n=16)$ eram do sexo feminino, e 42,9\% ( $n=12)$ do sexo masculino.

Em relação à faixa etária, $46,43 \%(n=13)$ dos pacientes do grupo placebo possuíram idade superior a 45 e inferior a 60 anos, $42,86 \%(n=12)$ apresentaram idade entre 60 e 80 anos e, apenas 10,71\% $(n=03)$ estiveram na faixa entre 30 e 45 anos, não existindo indivíduos com menos de 30 anos (tabela 1). No grupo suplementado, 46,43\% ( $n=13)$ dos pacientes apresentaram faixa etária superior a 60 e inferior a 80 anos; $35,71 \%(n=10)$, entre 45 e 60 anos; $10,71 \%(n=03)$, idade inferior a 30 anos e somente $7,14 \%(n=02)$ apresentaram idade entre 30 e 45 anos (tabela 1).

Quanto ao estadiamento, $50,00 \%(n=14)$ dos indivíduos de cada grupo pertenciam ao estádio III. No estádio II, havia $35,71 \%(n=10)$ de pacientes do grupo placebo e $21,43 \%(n=06)$ do grupo que recebeu o Agaricus sylvaticus. No estádio I, 14,29\% $(n=04)$ pertenciam ao grupo placebo e, 28,57\% $(n=08)$ ao grupo suplementado (tabela 1).

Observou-se, em ambos os grupos, que 7,14\% $(n=02)$ dos pacientes eram tabagistas. No grupo placebo, $17,86 \%(n=05)$ eram ex-tabagistas e $75,00 \%$ $(n=21)$ nunca fumaram. No grupo suplementado, $28,57 \%(n=08)$ eram ex-tabagistas e $64,29 \%(n=18)$ relataram nunca terem fumado (tabela 1 ).

Em relação ao etilismo, $17,86 \%(n=05)$ dos pacientes suplementados com Agaricus sylvaticus relataram ingestão de bebidas alcoólicas, enquanto no 
Rev bras Coloproct Abril/Junho, 2007
Qualidade de Vida de Pacientes com Câncer Colorretal em Uso de Suplementação Dietética com Fungos Agaricus Sylvaticus após Seis Meses de Segmento: Ensaio Clínico Aleatorizado e Placebo-Controlado Renata Costa Fortes e Cols. grupo placebo esse número alcançou 10,71\% $(n=03)$ (tabela 1).

Com relação à prática de atividade física, 7,14\% $(n=02)$ dos pacientes de cada grupo a praticavam (tabela 1). Após seis meses de tratamento, observouse aumento dos pacientes que aderiram à prática de atividade física para $21,43 \%(n=06)$ e $35,71 \%(n=$ 10) nos grupos placebo e Agaricus sylvaticus, respectivamente (tabela 2).

Ao longo do tratamento, averiguou-se diminuição da disposição, no grupo placebo, de $85,71 \%(n=$ 24) para $78,57 \%(n=22)$ e, $64,29 \%(n=18)$, após três e seis meses, respectivamente, com conseqüente aumento do cansaço/fadiga (tabela 2). O contrário foi observado no grupo suplementado, onde a taxa de disposição aumentou de $67,86 \%(n=19)$ para $85,71 \%(n$ $=24$ ) após três meses, mantendo esses valores ao final do tratamento (tabela 2).

No grupo placebo, dos pacientes com idade entre 31 e 45 anos, 66,70\% $(n=02)$ apresentavam-se bem dispostos no início do tratamento, enquanto $91,70 \%$ ( $n=11)$ também relataram boa disposição entre os pacientes com faixa etária entre 46 e 60 anos e entre 61 e 80 anos (figura 1). No grupo suplementado, $66,70 \%(n=02)$, dos pacientes com idade entre $20 \mathrm{e}$ 30 anos relataram boa disposição no início do tratamento, assim como $66,70 \%(n=02)$ dos pacientes entre 31 e 45 anos, $70,00 \%(n=07)$ dos pacientes com idade entre 46 e 60 anos e $66,70 \%(n=08)$ dos com idade entre 61 e 80 anos (figura 2).

Após três meses de acompanhamento, no grupo placebo, dos pacientes com idade entre 31 e 45 anos, $50,00 \%(n=01)$ relataram boa disposição, assim como $61,50 \%(n=08)$ dos pacientes com idade entre 46 e 60 anos e 100,00\% $(n=12)$ daqueles com idade entre 61 e 80 anos (figura 1). No grupo Agaricus sylvaticus, $66,70 \%(n=02)$ dos pacientes com idade entre 20 e 30 anos referiram boa disposição no mesmo período do tratamento, assim como 100,00\% $(n=03)$ dos pacientes com idade entre 31 e 45 anos, 70,00\% $(n=07)$ dos

Tabela 1 - Características da população estudada, $n=56$.

\begin{tabular}{|c|c|c|c|c|}
\hline \multirow{3}{*}{$\begin{array}{l}\text { Características } \\
\text { Sexo }\end{array}$} & \multicolumn{4}{|c|}{ Grupos } \\
\hline & \multicolumn{2}{|c|}{ Placebo $(n=28)$} & \multicolumn{2}{|c|}{ Agaricus sylvaticus $(n=28)$} \\
\hline & $n$ & $\mathrm{Fp}$ & $n$ & $\mathrm{Fp}$ \\
\hline Feminino & 16 & $57,10 \%$ & 16 & $57,10 \%$ \\
\hline Masculino & 12 & $42,90 \%$ & 12 & $42,90 \%$ \\
\hline \multicolumn{5}{|l|}{ Faixa etária } \\
\hline$<30$ anos & 00 & $0,00 \%$ & 03 & $10,71 \%$ \\
\hline$\geq 30$ e $<45$ anos & 03 & $10,71 \%$ & 02 & $7,14 \%$ \\
\hline$\geq 45$ e $<60$ anos & 13 & $46,43 \%$ & 10 & $35,71 \%$ \\
\hline$\geq 60$ e $<80$ anos & 12 & $42,86 \%$ & 13 & $46,43 \%$ \\
\hline \multicolumn{5}{|l|}{ Estadiamento } \\
\hline Estádio I & 04 & $14,29 \%$ & 08 & $28,57 \%$ \\
\hline Estádio II & 10 & $35,71 \%$ & 06 & $21,43 \%$ \\
\hline Estádio III & 14 & $50,00 \%$ & 14 & $50,00 \%$ \\
\hline \multicolumn{5}{|l|}{ Tabagismo } \\
\hline Nunca fumou & 21 & $75,00 \%$ & 18 & $64,29 \%$ \\
\hline Ex-tabagista & 05 & $17,86 \%$ & 08 & $28,57 \%$ \\
\hline Tabagista & 02 & $7,14 \%$ & 02 & $7,14 \%$ \\
\hline \multicolumn{5}{|c|}{ Ingestão de bebida alcoólica } \\
\hline Não-etilista & 25 & $89,29 \%$ & 23 & $82,14 \%$ \\
\hline Etilista & 03 & $10,71 \%$ & 05 & $17,86 \%$ \\
\hline \multicolumn{5}{|c|}{ Prática de atividade física } \\
\hline Não-sedentário & 02 & $7,14 \%$ & 02 & $7,14 \%$ \\
\hline Sedentário & 26 & $92,86 \%$ & 26 & $92,86 \%$ \\
\hline
\end{tabular}


Rev bras Coloproct Abril/Junho, 2007
Qualidade de Vida de Pacientes com Câncer Colorretal em Uso de Suplementação Dietética com Fungos Agaricus Sylvaticus após Seis Meses de Segmento: Ensaio Clínico Aleatorizado e Placebo-Controlado Renata Costa Fortes e Cols.

Tabela 2 - Principais sintomas relatados pelos pacientes dos grupos placebo e Agaricus sylvaticus.

\begin{tabular}{|c|c|c|c|c|c|c|c|c|c|c|c|c|}
\hline \multirow{3}{*}{$\begin{array}{l}\text { Sintomas } \\
\text { Sono }\end{array}$} & \multicolumn{6}{|c|}{ Placebo $(n=28)$} & \multicolumn{6}{|c|}{ Agaricus sylvaticus $(n=28)$} \\
\hline & \multicolumn{2}{|c|}{ Inicial } & \multicolumn{2}{|c|}{ Três meses } & \multicolumn{2}{|c|}{ Seis meses } & \multicolumn{2}{|c|}{ Inicial } & \multicolumn{2}{|c|}{ Três meses } & \multicolumn{2}{|c|}{ Seis meses } \\
\hline & $n$ & $\mathrm{Fp}$ & $n$ & $\mathrm{Fp}$ & $n$ & $\mathrm{Fp}$ & $n$ & $\mathrm{Fp}$ & $n$ & $\mathrm{Fp}$ & $n$ & $\mathrm{Fp}$ \\
\hline Dorme mal & 04 & $14,29 \%$ & 06 & $21,43 \%$ & $6 \quad 03$ & $10,71 \%$ & 02 & $7,14 \%$ & 02 & $7,14 \%$ & 01 & $3,57 \%$ \\
\hline Insônia & 02 & $7,14 \%$ & 04 & $14,29 \%$ & 08 & $28,57 \%$ & 03 & $10,71 \%$ & 03 & $10,71 \%$ & 02 & $7,14 \%$ \\
\hline Sem alterações & 22 & $78,57 \%$ & 18 & $64,29 \%$ & b 17 & $60,71 \%$ & 23 & $82,14 \%$ & 23 & $82,14 \%$ & 25 & $89,29 \%$ \\
\hline \multicolumn{13}{|l|}{ Disposição } \\
\hline Bem disposto & 24 & $85,71 \%$ & 22 & $78,57 \%$ & b 18 & $64,29 \%$ & 19 & $67,86 \%$ & 24 & $85,71 \%$ & 24 & $85,71 \%$ \\
\hline Cansaço/fadiga & 04 & $14,29 \%$ & 06 & $21,43 \%$ & $6 \quad 10$ & $35,71 \%$ & 09 & $32,14 \%$ & 04 & $14,29 \%$ & 04 & $14,29 \%$ \\
\hline \multicolumn{13}{|l|}{ Humor } \\
\hline Feliz/ satisfeito & 18 & $64,29 \%$ & & & 12 & $42,86 \%$ & 17 & $60,71 \%$ & & & 24 & $85,71 \%$ \\
\hline Nem feliz nem triste & 10 & $35,71 \%$ & & & 10 & $35,71 \%$ & 11 & $39,29 \%$ & & & 04 & $14,29 \%$ \\
\hline Infeliz/ insatisfeito & 00 & $00,00 \%$ & & & 06 & $21,43 \%$ & 00 & $00,00 \%$ & & & 00 & $00,00 \%$ \\
\hline \multicolumn{13}{|l|}{ Atividade Física } \\
\hline Sedentário & 26 & $92,86 \%$ & 23 & $82,14 \%$ & $6 \quad 22$ & $78,57 \%$ & 26 & $92,86 \%$ & 22 & $78,57 \%$ & 18 & $64,29 \%$ \\
\hline Não-sedentário & 02 & $7,14 \%$ & 05 & $17,86 \%$ & $6 \quad 06$ & $21,43 \%$ & 02 & $7,14 \%$ & 06 & $21,43 \%$ & 10 & $35,71 \%$ \\
\hline
\end{tabular}

pacientes com idade entre 46 e 60 anos e $100,00 \%$ ( $n$ $=12)$ dos pacientes com faixa etária entre 61 e 80 anos (figura 2).

Ao final do tratamento, observaram-se os seguintes resultados: no grupo placebo, $50,00 \%(n=01)$ dos pacientes com idade entre 31 e 45 anos apresentaram boa disposição, assim como 46,20\% $(n=06)$ dos pacientes entre 46 e 60 anos e $83,30 \%(n=10)$ dos pacientes com idade entre 61 e 80 anos (figura 1). No grupo Agaricus sylvaticus, $66,70 \%(n=02)$ dos pacientes com idade entre 20 e 30 anos relataram boa disposição, o mesmo ocorrendo com $66,70 \%(n=02)$ dos pacientes com idade entre 31 e 45 anos, $100,00 \%$ ( $n=$ 10) dos pacientes com idade entre 46 e 60 anos e $90,90 \%(n=10)$ dos pacientes com idade entre 61 e 80 anos (figura 2).

Entre os pacientes do grupo placebo, $14,29 \%$ $(n=04)$ relataram dormir mal no início do tratamento e $10,71 \%(n=03)$ referiram o mesmo após seis meses (tabela 2). No grupo Agaricus sylvaticus, $7,14 \%$ ( $n=$ $02)$ apresentaram essa queixa no início do tratamento, enquanto $3,57 \%(n=01)$ a apresentaram ao final (tabela 2).

Insônia foi relatada por $7,14 \%(n=02)$ do pacientes do grupo placebo e por $10,71 \%(n=03)$ do grupo suplementado no início do acompanhamento (tabela 2). Após seis meses, observou-se que $28,57 \%$ ( $n$

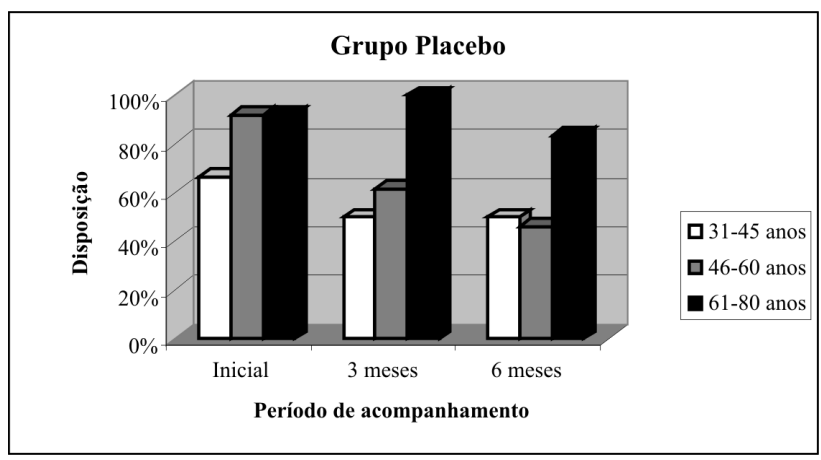

Figura 1 - Disposição dos pacientes do grupo placebo durante todo o período de acompanhamento.

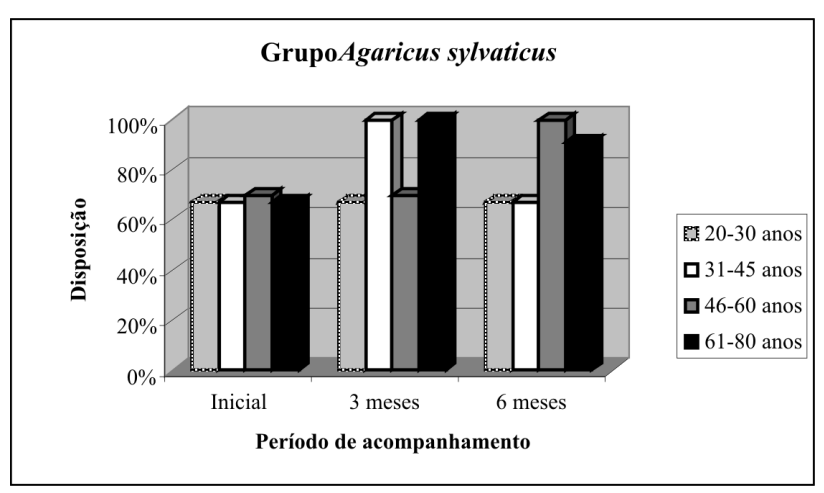

Figura 2 - Disposição dos pacientes do grupo Agaricus sylvaticus durante todo o período de acompanhamento. 
$=08)$ e, $7,14 \%(n=02)$ dos pacientes dos grupos placebo e Agaricus sylvaticus permaneceram com insônia, respectivamente (tabela 2 ).

Em relação ao humor, averiguou-se, no grupo placebo, que $64,29 \%(n=18)$ dos pacientes iniciaram o estudo sentindo-se felizes/satisfeitos e, $35,71 \%$ ( $n=$ 10) nem felizes/nem tristes. No grupo Agaricus sylvaticus, $60,71 \%(n=17)$ iniciaram o estudo sentindo-se felizes/satisfeitos e, 39,29\% $(n=11)$ nem felizes/nem tristes. Em ambos os grupos nenhum paciente relatou sentimento inicial de infelicidade/insatisfação (tabela 2).

Após seis meses de tratamento, os resultados observados foram os seguintes: dos $64,29 \%(n=18)$ dos pacientes do grupo placebo que apresentaram sentimento inicial de felicidade/satisfação, $16,67 \%(n=03)$ evoluíram para infelicidade/insatisfação, $27,78 \%(n=$ 05) para indiferença (nem felizes/nem tristes) e 55,55\% $(n=10)$ permaneceram com o mesmo humor inicial. No grupo Agaricus sylvaticus, dos $60,71 \%(n=17)$ dos pacientes felizes/satisfeitos, $88,24 \%(n=15)$ permaneceram com o mesmo humor inicial e apenas $11,76 \%(n=02)$ evoluíram para indiferença (tabela 2$)$.

Em relação aos $35,71 \%(n=10)$ dos pacientes do grupo placebo que iniciaram o estudo sentindo-se nem felizes/nem tristes, 30,00\% ( $n=03)$ evoluíram para infelicidade/insatisfação, $50,00 \%(n=05)$ permaneceram com o mesmo humor inicial e somente $20,00 \%$ ( $n$ = 02) evoluíram para felicidade/satisfação. Ao passo que, no grupo Agaricus sylvaticus, de 39,29\% ( $n=$ 11) dos pacientes que iniciaram o estudo com sentimento de indiferença, $81,82 \%(n=09)$ evoluíram para felicidade/satisfação e apenas $18,18 \%(n=10)$ não alteraram o humor (tabela 2).

Quanto à dor, no início do tratamento, 64,29\% $(n=18)$ dos pacientes do grupo placebo e $42,86 \%$ ( $n=$ 12) do grupo Agaricus sylvaticus queixaram-se de algum tipo de dor. Após seis meses, houve aumento dessa queixa para $71,43 \%(n=20)$ entre os pacientes do grupo placebo e uma redução para $32,14 \%(n=09)$ no grupo suplementado.

Quando as queixas de dores foram analisadas separadamente, observou-se, no grupo Agaricus sylvaticus, redução das dores retal, de 7,14\% $(n=02)$ para $3,57 \%(n=01)$, abdominal, de $14,29 \%(n=04)$ para $7,14 \%(n=02)$, e outras, de $21,43 \%(n=06)$ para $14,29 \%(n=04)$, após seis meses de suplementação. Ao passo que, no grupo placebo, observou-se aumento das mesmas, exceto da dor retal que permaneceu inalterada. Em relação à cefaléia, observou-se que a mesma aumentou nos dois grupos: de 3,57\% $(n=01)$ para $17,86 \%(n=05)$, no início e após seis meses, respectivamente, no grupo placebo e, de 3,57\% $(n=01)$ para $10,71 \%(n=03)$, no grupo Agaricus sylvaticus (figura 3).

\section{DISCUSSÃO}

Avanços tecnológicos no diagnóstico e tratamento precoces das neoplasias malignas têm aumentado a sobrevida dos pacientes e, conseqüentemente, maior ênfase tem sido dada a qualidade de vida desta população9.

$\mathrm{Na}$ qualidade de vida, entre outros aspectos, está incluída a fadiga como falta de motivação. A fadiga é altamente prevalente e é um sintoma debilitante e crônico em pacientes com câncer, sendo observada nesta população durante e após o tratamento adjuvante $^{9,10}$.

Pacientes oncológicos apresentam freqüentemente náuseas, vômitos, diarréia, diminuição de apetite e perda de peso, acrescidos de outros sintomas como dor e fadiga. Esses sintomas geralmente influenciam no desenvolvimento das atividades habituais como trabalhar, ter atividades de lazer, preparar as refeições, disposição física para interagir na sociedade, afetando significativamente os indicadores de qualidade de vida ${ }^{11}$.

Em indivíduos saudáveis, a fadiga é considerada um fator protetor, surgindo como resposta regulatória de estresse físico ou psicológico. Porém, nos pacientes com neoplasias malignas, a fadiga representa um sintoma desagradável e limitante para a realização de atividades diárias?.

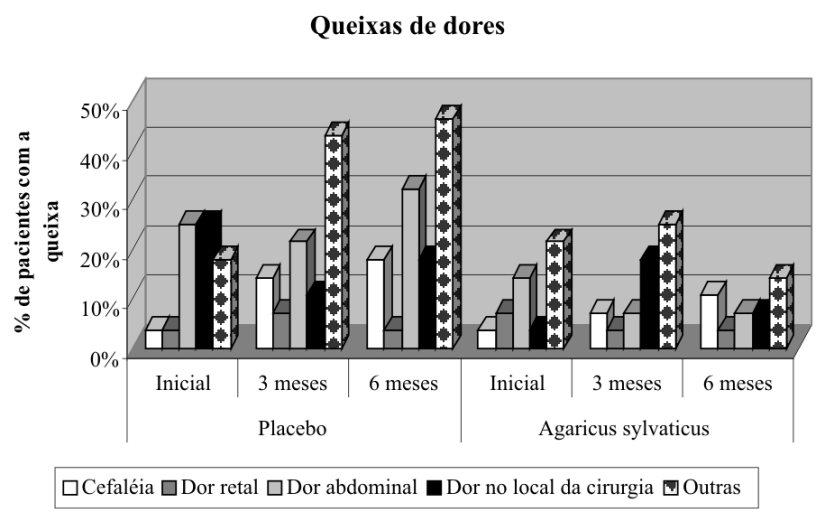

Figura 3 - Queixas de dores relatadas pelos pacientes nos diferentes grupos estudados. 
Antes do início do tratamento, nos grupos placebo e Agaricus sylvaticus deste estudo, apenas $7,14 \%$ dos pacientes praticavam alguma atividade física. Após seis meses, observou-se aumento da prática de atividade física em ambos os grupos, porém com maior percentual no grupo suplementado.

Observou-se, também, no grupo placebo, redução da disposição (de 85,71\% para 64,29\%) com conseqüente aumento do cansaço/fadiga (de 14,29\% para $35,71 \%$ ) durante todo o período de acompanhamento. Resultados inversos foram encontrados no grupo Agaricus sylvaticus com aumento da disposição (de $67,86 \%$ para $85,71 \%$ ) e redução do cansaço/fadiga (de $32,14 \%$ para $14,29 \%$ ) após três meses de suplementação, mantendo-se esses resultados até o final do tratamento.

Ao se fazer a correlação entre disposição e idade, averiguou-se, em ambos os grupos, que os indivíduos na faixa etária inferior a 46 anos apresentaram menor disposição quando comparados com os pacientes mais velhos. Porém, ao analisar cada faixa etária separadamente, observou-se, no grupo Agaricus sylvaticus, após seis meses de suplementação, aumento da disposição nos pacientes com idade superior a 30 anos e, manutenção da disposição naqueles entre 20 e 30 anos. No grupo placebo, os resultados encontrados foram contraditórios, demonstrando redução da taxa de disposição em todas as faixas etárias.

Estudos têm comprovado que a idade exerce influência significativa sobre a reação psicológica ao diagnóstico do câncer e ao tratamento antineoplásico, ou seja, pacientes mais jovens, além do maior impacto psicológico, geralmente apresentam menor grau de amadurecimento, referem mais dificuldades econômicas e maior número de dias perdidos no trabalho quando comparado com os mais velhos ${ }^{1}$.

Evidências científicas demonstram que a fadiga pode persistir por meses ou anos após completar o tratamento com sucesso em uma minoria de pacientes. A fadiga no câncer é multifatorial e alguns mecanismos promissores têm sido identificados, incluindo distúrbios do sono, estresse psicológico, dor, entre outros sintomas, podendo envolver de forma severa os domínios mental, físico e emocional ${ }^{12,13}$.

Observou-se, neste estudo, no grupo Agaricus sylvaticus, redução da insônia (de $10,71 \%$ para $7,14 \%$ ) e do hábito de dormir mal (de 7,14\% para 3,57\%) após seis meses de suplementação. Esses resultados não foram encontrados no grupo placebo.
A progressão do câncer e dos sintomas associados está intimamente relacionada a quadros depressivos, podendo refletir em aumento do prejuízo funcional com conseqüente piora da qualidade de vida. Tristeza, raiva, ansiedade e angústia são respostas emocionais negativas freqüentemente observadas em pacientes com câncer que, quando intensas e duradouras, podem culminar com o diagnóstico de depressão ${ }^{13}$.

Pesquisas científicas têm demonstrado que pacientes deprimidos apresentam um risco três vezes maior de não-adesão ao tratamento quando comparados com indivíduos isentos de sintomas depressivos ${ }^{13}$. Sendo assim, o diagnóstico de depressão em pacientes com câncer baseia-se em sintomas como desânimo, insônia, dificuldade de cooperar com o tratamento, sentimentos de menos-valia, culpa e morte, perda da esperança, falta de prazer e de apego pela vida, devendo ser detectado o mais precocemente possível ${ }^{14}$.

No presente estudo, observou-se, no grupo Agaricus sylvaticus, uma melhora no estado do humor de felicidade/satisfação (de $60,71 \%$ para $85,71 \%$ ) com consequiente redução do sentimento de indiferença (de 39,29\% para 14,29\%), após seis meses de suplementação. Esses resultados não foram encontrados no grupo placebo que apresentou redução do sentimento de felicidade/satisfação (de 64,29\% para $42,86 \%$ ) e aumento da infelicidade/insatisfação (de $0,00 \%$ para $21,43 \%$ ).

Estudos evidenciam, em pacientes com câncer, que os sintomas depressivos podem ocorrer devido a diversos fatores como falta de regulação do eixo hipotálamo-pituitária-adrenal e do eixo tireoideano, oscilações na função imune das células Natural Killer (NK), redução da secreção do hormônio de crescimento, alteração do ritmo circadiano, presença de alterações metabólicas, além do uso de medicações específicas como corticóides, tamoxifeno e interferon-alfa (INF- $\alpha$ ). A ativação de citocinas pró-inflamatórias como as interleucinas $1 \mathrm{e}$ 6 (IL-1 e IL-6) também são capazes de induzir modificações na função neuronal e promover ativação do eixo hipotálamo-pituitária-adrenal ${ }^{13}$.

Os polissacarídeos presentes nos fungos medicinais são capazes de exercer efeito no organismo humano através do aumento das funções imunológicas, estimulação da ativação de células NK, linfócitos T, linfócitos $\mathrm{B}$ e células complementares, promovendo um 
aumento do número de macrófagos e monócitos, além da proliferação e/ou produção de anticorpos e de várias citocinas como as IL-2 e IL-6, INF- $\alpha$, fator de necrose tumoral e, dessa forma, evitar a regeneração e a metástase do câncer ${ }^{4}$.

O câncer está freqüentemente associado com a dor que, por sua vez, afeta a afetividade, a função, o apetite, o sono, provoca depressão, além de aumentar o risco de suicídio. Pacientes com câncer podem apresentar associação de dores crônicas e distúrbios afetivos. $58 \%$ a $80 \%$ dos pacientes adultos oncológicos internados sofrem de dor, sendo que as dores moderadas e intensas estão presentes em $30 \%$ a $40 \%$ nos estágios intermediários e, cerca de, $87 \%$ nas fases mais avançadas ${ }^{15}$.

Em 64,29\% pacientes do grupo placebo e, $42,86 \%$ dos pacientes pertencentes ao grupo Agaricus sylvaticus queixaram-se de dores antes do início do tratamento. Após seis meses, observouse aumento dessa queixa para $71,43 \%$ no grupo placebo e redução para $32,14 \%$ no grupo suplementado.

Ao analisar as queixas de dores separadamente, observou-se, no grupo Agaricus sylvaticus, redução das dores retal, abdominal e outras, após seis meses de suplementação. Ao passo que, no grupo placebo, observou-se aumento das mesmas, exceto da dor retal que permaneceu inalterada neste período. A cefaléia aumentou em ambos os grupos durante todo o período de acompanhamento, sendo este aumento maior no grupo placebo.

Um estudo comprovou que a administração parenteral de proteoglucanas extraídas de fungos medicinais aliada à quimioterapia foi capaz de prolongar o tempo de sobrevida, restaurar os parâmetros imunológicos e melhorar a qualidade de vida de pacientes com câncer de cólon e outros carcinomas em comparação aos pacientes tratados apenas com quimioterapia ${ }^{16}$.

Outro estudo demonstrou que a suplementação dietética com fungos Agaricus sylvaticus em pacientes com câncer colorretal em tratamento quimioterápico por um período de três meses, foi capaz de promover melhora significativa na disposição em 55\% dos sujeitos, ausência de alterações em $36 \%$ e, apenas $9 \%$ relatara desânimo. No grupo placebo, estas alterações não foram observadas onde $28 \%$ dos pacientes apresentaram tontura, $27 \%$ dores, $18 \%$ insônia, $9 \%$ fraqueza e, $18 \%$ ausência de alterações ${ }^{8}$.
Está bem esclarecida na literatura a presença de diversas alterações de humor, motoras, somáticas, sociais, cognitivas, entre outras em pacientes com câncer. Todas essas alterações incluem tristeza, perda de interesse por qualquer tipo de atividade, falta de prazer, crises de choro, modificações do humor, inibição ou retardo dos movimentos e agitação, alterações no sono, variações do apetite, fadiga, apatia, incapacitação para o desempenho das tarefas cotidianas, desesperança, desamparo, idéias de culpa e de suicídio, indecisão, ansiedade, irritabilidade, entre outras ${ }^{2}$.

No presente estudo, observou-se, no grupo suplementado, melhoria de praticamente todos os indicadores avaliados, principalmente quando comparados com o grupo placebo, demonstrando a possível presença de substâncias bioativas no fungo Agaricus sylvaticus capazes de melhorar a qualidade de vida desses pacientes.

Estudos evidenciam a capacidade das proteoglucanas extraídas de fungos medicinais agirem através do aumento significativo da sobrevida de pacientes com diversos tipos de câncer, incluindo o colorretal, além de melhorar a qualidade de vida e promover alívio dos sintomas em $70 \%$ a $97 \%$ dos pacientes com neoplasias malignas ${ }^{4,17,18}$. Acredita-se que o provável mecanismo de ação está relacionado à estimulação das funções imunológicas, da atividade fagocitária dos macrófagos e melhoria das funções do sistema retículo-endotelial ${ }^{4}$.

Além das proteoglucanas, diversas outras substâncias bioativas estão presentes nos fungos medicinais como glucanas, lectinas, ergosterol, triterpenos, arginina, glutamina, entre outras. Os mecanismos de ação dessas substâncias ainda não estão completamente elucidados na literatura, mas evidências científicas sugerem que esses compostos bioativos são capazes de modular o processo de carcinogênese nos estágios de iniciação, promoção e progressão e, dessa forma, promover benefícios adicionais aos portadores de diversos tipos de câncer ${ }^{4}$.

\section{CONCLUSÃO}

Os resultados sugerem que a suplementação dietética com fungos Agaricus sylvaticus é capaz de melhorar a qualidade de vida de pacientes com câncer colorretal em fase pós-operatória por reduzir significativamente os efeitos deletérios ocasionados pela própria enfermidade e pelo tratamento convencional da mesma. 
Rev bras Coloproct Abril/Junho, 2007
Qualidade de Vida de Pacientes com Câncer Colorretal em Uso de Suplementação Dietética com Fungos Agaricus Sylvaticus após Seis Meses de Segmento: Ensaio Clínico Aleatorizado e Placebo-Controlado Renata Costa Fortes e Cols.

ABSTRACT: Introduction: Gastrointestinal cancer jeopardizes the quality of life through physiological, metabolic and psychological alterations. Medicinal fungus may boost the quality of life of patients with cancer. Objective: To evaluate the effects of the dietary supplementation with Agaricus sylvaticus fungus in relation to the quality of life in patients with colorectal cancer during post-surgery phase. Methodology: Randomized, double-blind, placebo-controlled clinical trial carried out at the Federal District Hospital - Brazil, for six months. Samples of 56 enrolled patients ( 24 men and 32 women), stadiums phase I, II and III, separated as placebo and Agaricus sylvaticus $(30 \mathrm{mg} / \mathrm{kg} / \mathrm{day})$ supplemented groups: In order to evaluate indicators for quality of life, it was used form-standard and direct anamnese-standard. The method of analysis for results was qualitative and descriptive, using the Microsoft Excel 2003 and Epi Info 2004 programs. Results: After six months of treatment, it was observed an increase of adhesion to physical activity; improved disposition and good mood, reduction of complaints, pains, and alterations of sleep such as insomnia and bad nights of sleep when Agaricus sylvaticus and placebo groups were compared. Conclusion: The results suggest that the dietary supplementation with Agaricus sylvaticus may improve the quality of life of patients with colorectal adenocarcinoma in post-surgery phase.

Keywords: Agaricus sylvaticus fungus, quality of life, colorectal cancer, metabolic alterations, physiologic alterations.

\section{REFERÊNCIAS}

1. Conde DM, Pinto-Neto AM, Júnior RF, Aldrighi JM. Qualidade de vida de mulheres com câncer de mama. Rev Bras Ginecol Obstet 2006; 28(3): p 195-204.

2. Thompson C. Affective disordes. In: Thompson C, editor. The instruments of psychiatric research. London: John Wiley \& Sons; 1989. p 87-126.

3. Novaes MRCG; Fortes RC. Efeitos antitumorais de cogumelos comestíveis da família agaricaceae. Rev Nutr Bras 2005; 4(4): p 207-17.

4. Fortes RC, Novaes MRCG. Efeitos da suplementação dietética com cogumelos Agaricales e outros fungos medicinais na terapia contra o câncer. Rev Bras Cancerol 2006; 52(4): p 363-71.

5. Novaes MRCG, Novaes LCG. Fármaco-nutrientes em cogumelos comestíveis Agaricales e outros basidiomicetos. Rev Bras Nutr Clin 2005; 20(3): p 181-7.

6. Fortes RC, Taveira VC, Novaes MRCG. The immunomodulator role of $\beta-\mathrm{D}$-glucans as co-adjuvant for cancer therapy. Rev Bras Nutr Clin 2006; 21(2): p 163-8.

7. Novaes MRCG, Fortes RC, Recôva V, Melo A. Effects of nutritional supplementation with Agaricus sylvaticus (Cogumelo do Sol) in post-surgery patients with adenocarcinoma of gross intestine. Proceedings of the 27 th International Congress of ESPEN and Clinical Nutrition; 2005 Aug 27-30; Brussels, Belgium: Elsevier 2005. p. 672.

8. Fortes RC, Novaes MRCG, Recôva V, Melo A. Quality of life during dietary supplementation with Agaricus sylvaticus in patients with adenocarcinoma of gross intestine in postsurgical chemotherapy treatment. Proceedings of the I World Congress of Public Health Nutrition/ VII National Congress of the Spanish Society of Community Nutrition and Public Health Nutrition; 2006 September 28-30; Barcelona, Spain: NS 2006. p. 237.
9. Servaes P, Verhagen S, Bleijenber G. Fatigue in cancer patients during and after treatment: prevalence, correlates and interventions. Eur J Cancer 2002; 38 (1): p 27-43.

10. Flechtner H, Bottomley A. Fatigue and quality of life: lessons from the real world. The oncologist 2003; 8(suppl 1): p 5-9.

11. Baxter YC, Waitzberg DL. Qualidade de vida do paciente oncológico. In: Waitzberg DL. Dieta, nutrição e câncer. São Paulo: Atheneu. 2004. p 573-80.

12. Bower JE. Prevalence and causes of fatigue after cancer treatment: the next generation of research. Journal of Clinical Oncology 2005; 23(33): p 8280-82.

13. Calil HM, Fernandes FN, Scippa AM, Quarantini L. Depressão em pacientes com câncer: diagnóstico e tratamento. Câncer Hoje 2006; 4(14): p 4-7.

14. Lourenço MTC, Costa CL. Aspectos psicossociais do paciente com câncer. In: Ikemori EHA, editores. Nutrição em oncologia. São Paulo: Marina e Tecmedd, 2003. p. 369-74

15. Júnior JOO, Martins IMSM. Dor oncológica. In: Ikemori EHA, editores. Nutrição em oncologia. São Paulo: Marina e Tecmedd, 2003. p. 376-401.

16. Lindequist U, Niedermeyer THJ, Jülich WD. The pharmacological potential of mushrooms. eCAM 2005; 2(3): p 285-99.

17. Didukh MY, Wasser SP, Nevo E. Medicinal value of species of the family Agaricaceae cohn (higher basidiomycetes) and future perspectives. International Journal of Medicinal Mushrooms 2003; 5(2): p 133-52.

18. Sullivan R, Smith JE, Rowan NJ. Medicinal mushrooms and cancer therapy. Spring 2006; 49(2): p 159-70.

Endereço para correspondência:

RENATA COSTA FORTES

QI 14. Conjunto J. Casa 26.

Guará 1 / DF - CEP: 71.015-100.

Telefone: (0xx61) 9979-9463

E-mail: renatacfortes@yahoo.com.br 